\title{
Failed retinal surgery caused by intraocular gas injection
}

\author{
P B JOHNSTON, C J F MAGUIRE, AND W C LOGAN
}

From the Department of Ophthalmology, Eye and Ear Clinic, Royal Victoria Hospital, Belfast BT12 6BA, Northern Ireland.

SUMMARY Four patients developed inferior retinal redetachment following initially successful surgery which included intraocular injection of air and sulphahexafluoride (SF6) mixture. In each case the intravitreal gas bubble produced vitreoretinal traction which opened inferior retinal breaks and led to retinal separation. Cautious use of intraocular gas is advised when bullous upper rhegmatogenous retinal detachments are accompanied by inferior retinal breaks.

The use of intravitreal air injection to achieve internal closure of retinal breaks was advocated by Rosengren in 1938.' More recently Norton ${ }^{2}$ and Gilbert and $\mathrm{McLeod}^{3}$ described surgical procedures with air or sulphur hexafluoride $\left(\mathrm{SF}_{6}\right)$ that overcome the difficulties presented by bullous rhegmatogenous retinal detachment and fish-mouthing of large equatorial retinal breaks. These techniques have proved reliable. However, our recent experience suggests that in certain cases intraocular gas may reverse initially successful reattachment surgery. We describe four patients who developed inferior retinal redetachment following uneventful retinal surgery which included intraocular injection of gas.

Correspondence to P B Johnston, FRCS.

\section{Case reports}

\section{CASE 1}

A 71-year-old man developed a deep upper-half retinal detachment in his right eye. One large Ushaped tear was situated at the upper temporal equator, and three small flap tears were found at the inferior temporal quadrant within attached retina (Fig. 1, A). A retinal detachment procedure was performed with a piece of solid silicone (287 tire) explant and encircling 240 band. Before scleral buckling, subretinal fluid was drained and the ocular volume restored with $1 \mathrm{ml} \mathrm{SF}_{6}$ gas injected into the vitreous cavity. The retinal breaks were surrounded by cryo application. The retina was attached at the
Fig. 1A Superior bullous retinal detachment. Inferior retina contains three flat tears (B). Gas bubble holds the superior retinal break and buckle in apposition, but subretinal fluid has collected below.
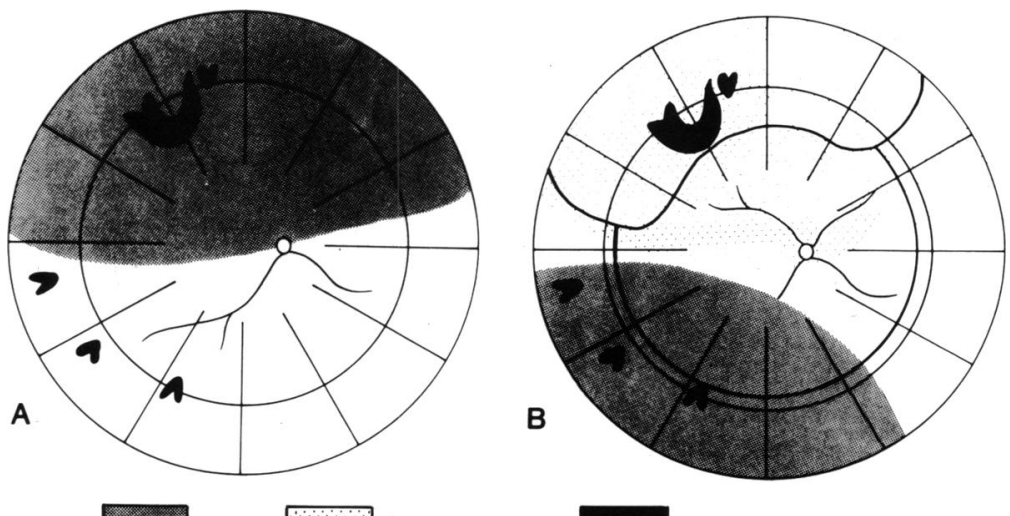

R D 322 

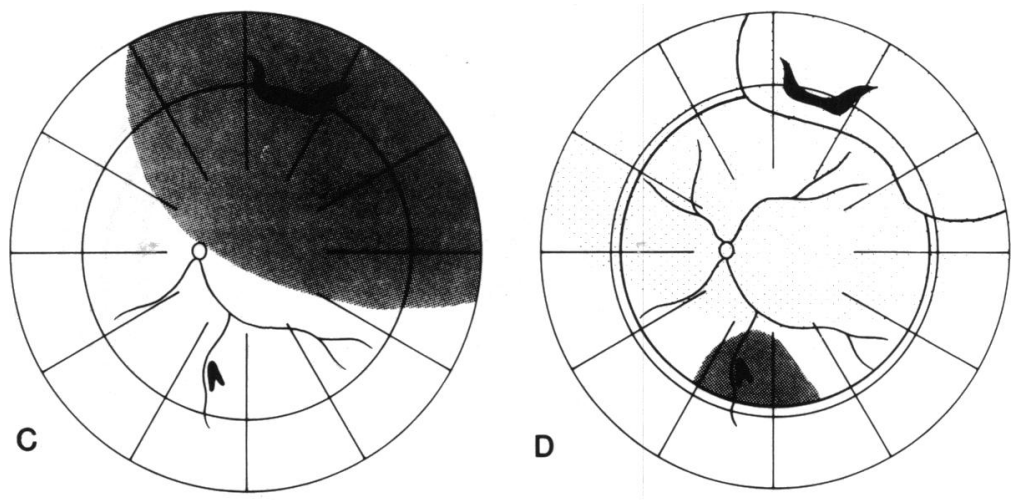

Retinal Break
Fig. 2C Largesuperior balloon detaciment. The inferior retina contains one flat postequatorial tear (D). Gas bubble holds the superior break and buckle in apposition, while subretinal fluid collects around the inferior break. first postoperative day, but by the second day there was an inferior bullous retinal detachment associated with the three lower tears (Fig. 1, B). The intravitreal gas bubble opened the inferior tears widely by increasing vitreoretinal traction when the patient was erect. The silicone tire was extended circumferentially to cover these tears, and subretinal fluid was released. The retina reattached but three weeks later developed total detachment with widespread changes of proliferative vitreoretinopathy.

\section{CASE 2}

A 59-year-old woman was referred with a superior balloon retinal detachment in her left eye. The detached retina contained a large U-shaped tear, and the inferior retina was attached with one postequatorial flap tear (Fig. 2, C). A retinal detachment procedure was performed with a broad circumferential buckle, encircling band 240 , and incomplete drainage of subretinal fluid. Cryotherapy was applied to the retinal breaks, and a scleral buckle was not considered necessary for the inferior break. After the operation there was residual subretinal fluid, and the upper retinal break remained elevated with a typical fish-mouth appearance. Five days later $0.5 \mathrm{ml}$ of pure $\mathrm{SF}_{6}$ gas was injected into the vitreous cavity, and the following day the superior retina was flattened on the buckle. Three days later subretinal fluid collected round the inferior retinal break, which was held open by transvitreo-retinal traction enhanced by movement of the intravitreal gas bubble (Fig. 2, D). The inferior break was successfully closed with a $7 \mathrm{~mm}$ radial sponge.

CASE 3

A 50-year-old woman developed a bullous detachment of the left superior retina which contained two U-shaped tears and three round holes (Fig. 3, E). The lower retina was attached with pre-equatorial lattice degeneration at the inferior periphery. After external drainage of subretinal fluid the retina was repositioned with $2 \mathrm{ml}$ of $20 \% \mathrm{SF}_{6}$ gas and air mixture, which was injected into the vitreous cavity through the pars plana. The retinal breaks were

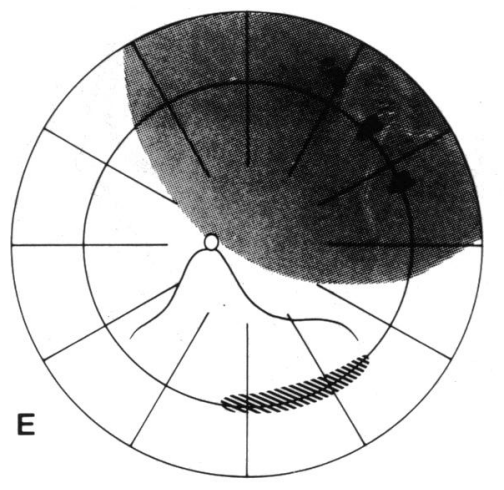

R D

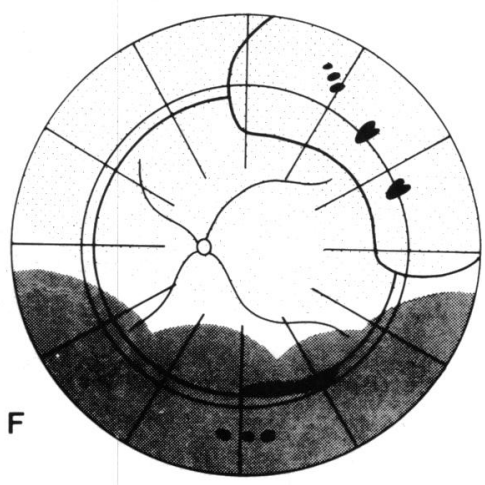

Retinal Break
Fig. 3E Superior balloon detachment. Inferior retina contains visible lattice degeneration (F). Gas bubble holds the superior retina and buckle in apposition. Three new tears resulted in inferior retinal detachment. 
treated with cyro application. The retinal breaks were buckled with a piece of solid silicone tire (287) and encircling band 240 . On the first postoperative day the retina was attached; on day 2 an inferior bullous retinal detachment developed with no identifiable retinal break. The circumferential buckle was extended to cover the inferior retinal periphery; however, this proved unsuccessful. Examination revealed fresh inferior retinal breaks (Fig. 3, F) which were held open by vitreous traction even though the scleral buckle was substantial. A third surgical procedure consisted of vitrectomy, internal gas/fluid exchange, and argon laser endophotocoagulation, which achieved reattachment of the retina.

\section{CASE 4}

A 67-year-old man developed a bullous detachment of the left superior retina which contained two Ushaped tears. After external drainage of subretinal fluid the pars plana was penetrated with a 27 gauge needle and the ocular volume restored with $1.5 \mathrm{ml}$ of $20 \% \mathrm{SF}_{6}$ gas and air mixture, which was injected into the vitreous gel. The retinal breaks were treated by cryo application and supported on a broad buckle and encircling band. On the first postoperative day the retina was reattached. On day 3 the inferior retina was elevated by vitreous traction, which increased when the intraocular gas bubble was displaced superiorly. Examination revealed three small inferior breaks at the posterior vitreous base. The retina attached after a second surgical procedure which comprised vitrectomy and rebuckling.

\section{Discussion}

Rosengren' introduced the method of intravitreal air injection for rhegmatogenous retinal detachment. This has been modified by many surgeons ${ }^{2-5}$ to combine scleral buckling, subretinal fluid drainage, and intravitreal air or $\mathrm{SF}_{6}$ gas injection for difficult cases such as bullous retinal detachments with large equatorial breaks. Stanford and $\mathrm{Chignell}^{6}$ reported an unacceptable incidence of failure of these cases where a technique of non-drainage or drainage without intravitreal gas injection is used. The deeply separated retina prevents accurate localisation and controlled cryotherapy of the retinal breaks. When subretinal fluid is drained, the retina settles on the buckle in folds, which creates the classical fish-mouth appearance. Many of these difficulties are avoided by preoperative reduction in the height of retinal detachment by means of an inferior rectus fixation suture. ${ }^{7}$ When immobilisation of the globe does not produce settling of the retinal balloon, a technique of subretinal fluid drainage, intravitreal air injection, cryopexy, and episcleral explant (D-ACE) ${ }^{3}$ is

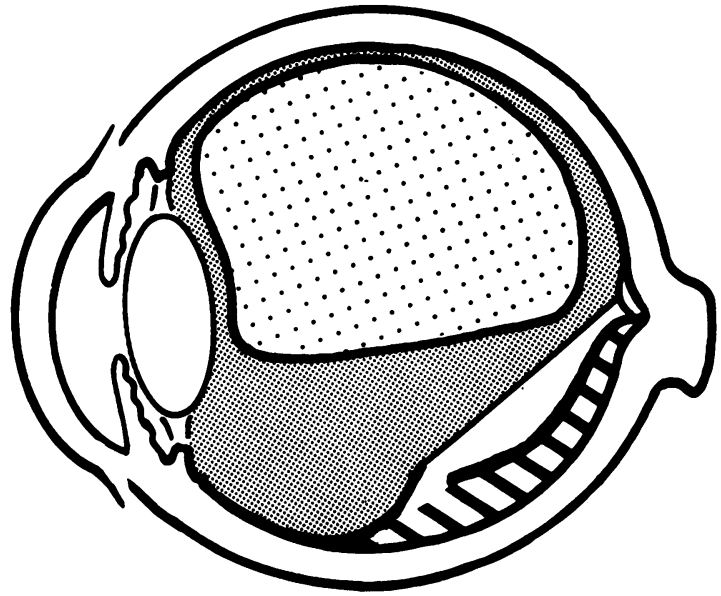

Fig. 4 Superior intragel gas bubble produces vitreous traction on inferior retinal break by stretching the posterior hyaloid.

employed. Using this technique for bullous retinal detachment Stanford and Chignell ${ }^{6}$ and Gilbert and McLeod $^{3}$ reported surgical success rates of $96 \%$ and $90 \%$ respectively. However, our four patients developed retinal redetachment, which we believe was related to the presence of intraocular gas. Two patients had pre-existing inferior retinal breaks within attached retina, and one had widespread lattice degeneration of the lower retina. All four patients developed opening of inferior retinal breaks and retinal separation following the use of intraocular gas, and in two cases fresh breaks were

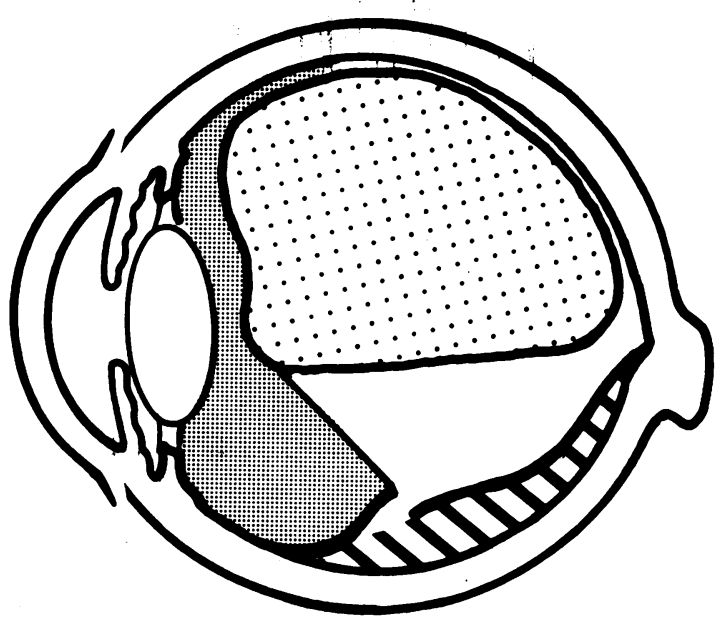

Fig. 5 Superior displacement of gas bubble into the retrohyaloid space creates inferior vitreous traction on the retinal break by anterior movement of the posterior hyaloid. 
detected following the first operation. Three patients were successfully treated by reoperation consisting of vitrectomy and intravitreal gas tamponade in two cases and rebuckling in the other. One patient developed proliferative vitreoretinopathy after a second rebuckling procedure, and further surgery was refused.

Gilbert and McLeod ${ }^{3}$ and Stanford and Chignell ${ }^{6}$ both report that intraocular gas injection has few serious complications, which mostly comprise subretinal haemorrhage at the subretinal fluid drainage site. However, Dreyer ${ }^{8}$ has described a patient who developed fresh inferior retinal breaks following retinal surgery which included intraocular injection of pure $\mathrm{SF}_{6}$ gas. Our four patients are comparable to Dreyer's case, and we suggest that superior displacement of the intraocular gas bubble caused increased vitreoretinal traction at the site of inferior retinal breaks and vitreous base. The proposed mechanism is due to stretching of the posterior hyaloid by the superiorly positioned gas bubble and can occur where the gas bubble is within the vitreous gel (Fig. 4) or has migrated into the retrohyaloid space (Fig. 5).

We agree that the D-ACE technique is valuable for difficult bullous detachments. However, caution should be exercised in the presence of preoperative inferior retinal breaks or lattice degeneration. Internal gas tamponade is necessary only for a short postoperative period to close superior retina breaks. When inferior retinal breaks exist prior to surgery, we believe it is advisable to use air rather than $\mathrm{SF}_{6}$ gas for internal tamponade of superior breaks. The delayed absorption and postoperative expansion ${ }^{2}$ of $\mathrm{SF}_{6}$ gas may increase and prolong vitreoretinal traction, which opens or creates inferior retinal breaks.

\section{References}

1 Rosengren B. Cases of retinal detachment treated with diathermy and injection of air into the vitreous body. Acta Ophthalmol (Kbh) 1938; 16: 573-9.

2 Norton EWD. Intraocular gas in the management of selected retinal detachments. Ophthalmology 1973; 77: 85-98.

3 Gilbert C, McLeod D. D-ACE surgical sequence for selected bullous retinal detachments. Br J Ophthalmol 1985; 69: 733-6.

4 Chawla HB, Colciro JA. Retinal detachment treated with intravitreal air: an evaluation of 241 cases. Br J Ophthalmol 1977; 61: 588-92.

5 McLean EB, Norton EWD. Use of intraocular air and sulfur hexafluoride gas in the repair of selected retinal detachments. Mod Probl Ophthalmol 1974; 12: 428-31.

6 Stanford MR, Chignell AH. Surgical treatment of superior bullous rhegmatogenous retinal detachments. Br J Ophthalmol 1985; 69: 729-32.

7 Johnston PB, Maguire CJE, Logan WC. Management of superior half bullous retinal detachment. Br J Ophthalmol 1981; 65: 618-22.

8 Dreyer RF. Sequential retinal tears attributed to intraocular gas. Am J Ophthalmol 1986; 102: 276-8.

Accepted for publication 5 March 1987. 